\title{
Wie das Moosglöckchen zu seinem Namen kam: Geschichten und Gedanken zum 300. Geburtstag von CARL vON Linné Teil 2
}

\author{
Karin Steinecke
}

\begin{abstract}
In 2007 the tercentenary of the birth of CARL VON LINNÉ is celebrated. The article describes scientific work and heritage of LinNé. It explains how the Twinflower got its scientific name Linnaea borealis.
\end{abstract}

\section{Zusammenfassung}

Im Jahr 2007 wird der 300. Geburtstag von CARL vON LinNÉ gefeiert. Der Beitrag beschreibt sein wissenschaftliches Werk sowie sein Erbe. Zudem wird erläutert, wie das Moosglöckchen den Namen Linnaea borealis bekam.

\section{LinNÉs Werk}

Der Lebenslauf von LinNé zeigt einen Aufstieg von einem armen Pfarrerssohn mit schlechten schulischen Leistungen zu einem Naturwissenschaftler von Weltruhm (vgl. Steinecke 2007). Dass LinNÉ bereits zu Lebzeiten besonders im Ausland große Anerkennung erfuhr und im eigenen Land in den Adelsstand erhoben wurde, war für die damalige Zeit keineswegs üblich. Viele Naturwissenschaftler jener Zeit sind erst posthum für ihre Ideen und Werke geehrt worden und hatten es zu Lebzeiten schwer, verstanden und anerkannt zu werden. Was also hat nun LinNé so Bahnbrechendes erdacht und erforscht und warum wurde er auf Anhieb so berühmt?

\section{LINNÉ als Naturwissenschaftler}

LinNÉ gilt als der Begründer der modernen Systematik, dem so genannten LINNÉ'schen System. Er entwickelte hierarchisch gegliederte Klassifizierungssysteme, von denen heute nur noch Spuren gültig sind, weil sie nicht auf genetischen Verwandtschaftsbeziehungen beruhen. Zusätzlich führte er die heute gebräuchliche binäre Nomenklatur ein, nach der alle Organismen in einem einheitlichen System mit einer Doppelbezeichnung wissenschaftlich benannt werden. Schließlich schuf LINNÉ eine einheitliche botanische Fachsprache, indem er unzählige Fachausdrücke genau definierte und erläuterte. Das LinNÉ’sche System und die binäre Nomenklatur wurden in Wissenschaftskreisen gern aufgenommen, nachdem das 17. Jahrhundert mit seinen naturwissenschaftlichen Ent- deckungen zu neuen Beschreibungen vieler Tier- und Pflanzenarten geführt hatte. LinNÉs System und Nomenklatur waren überschaubar, konsequent, leicht anwendbar sowie offen für die Integration neuer Taxa und setzten sich daher sehr schnell durch. Auch wenn Linné mit der Entwicklung seines Systems Großes geleistet hat, darf nicht vergessen werden, dass er keineswegs der erste Wissenschaftler war, der eine Klassifikation der Organismen versuchte. LinNé bezeichnete sich selbst nur als Reformator und nicht als Begründer der Systematik.

Wichtigste Vorgänger LinNÉs waren insbesondere John Ray (1627-1705) und Joseph Pitton De Tournefort (1656-1708), die bereits die Grundlagen für eine Klassifikation des Pflanzenreichs und - im Falle RAYs - teilweise auch der Insekten lieferten und deren Benennungen (u.a. die hierarchische Gliederung in Klassen, Gattungen und Arten) zum größten Teil von Linné übernommen wurden. TourneFORT teilte Pflanzen anhand ihres Blütenbaus ein. Gattungen wurden überwiegend aufgrund der Früchte beschrieben und Arten wurden durch verschiedenste Merkmale der ganzen Pflanze definiert.

Schon in seiner ersten Abhandlung, „Preluda Sponsaliorum Plantarum“ (1730) gelangte LinNé zu der Ansicht, dass sich Merkmale der Blüten mit Blütenblättern, Staubblättern und Stempel gut als Basis für eine Klassifikation eignen. Diese Vorgehensweise wurde von einigen Zeitgenossen als anstößig empfunden und heftig kritisiert. Dennoch blieb Linné diesem Ordnungsprinzip konsequent treu. Im „Systema 

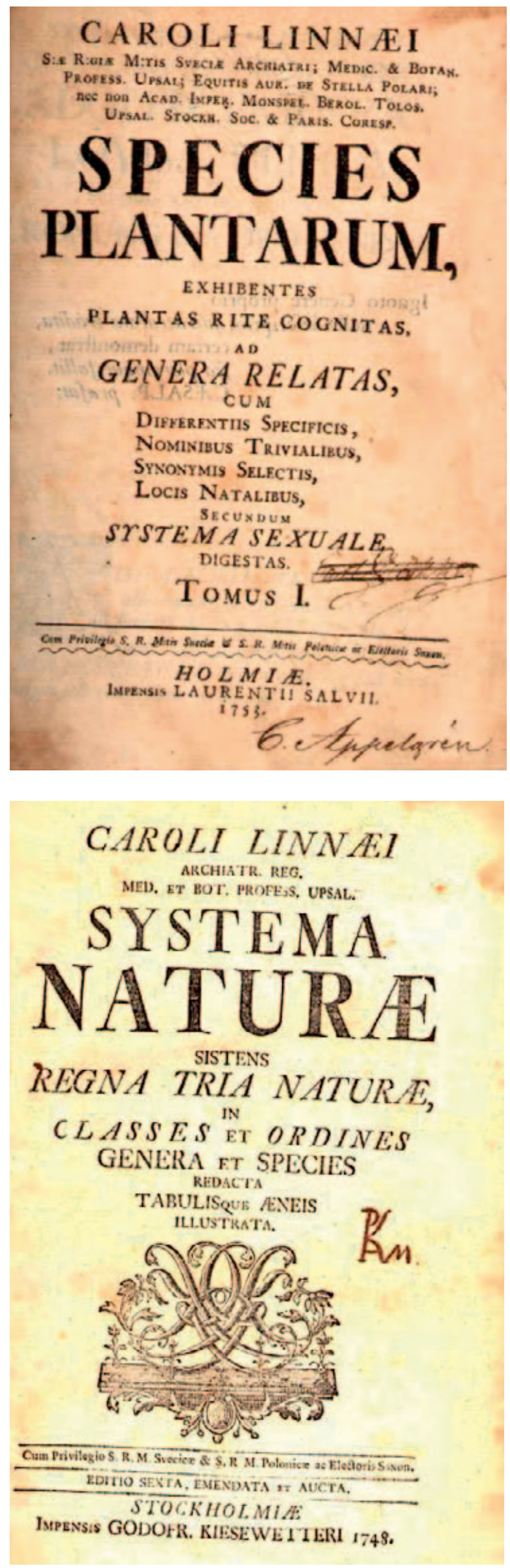

Naturae" ordnete er alle ihm bekannten Pflanzen, später auch Tiere und Mineralien seinem System zu, in der 1. Auflage (1730) noch auf 10, in der 13. Auflage (1770) bereits auf mehr als 3000 Seiten.

In seinem Werk „Species Plantarum“ (1753) führte LINNÉ für die Pflanzen die heute noch gültige binäre Nomenklatur ein. Seine einfachen wissenschaftlichen Doppelnamen der Arten aus Gattungsbezeichnung und Art-Epitheton lösten die bis dahin oft umständlichen, über mehrere Zeilen reichenden diagnostischen Artbenennungen ab. So hieß beispielsweise Physalis angulata, eine Verwandte der Andenbeere, früher Physalis annua ramosissima, ramis angulosis glabris, foliis dentatoserratis. Für die Ackerwinde, Convolvulus arvensis, war der Name Convolvulus foliis subrotundis, caule repente gebräuchlich. In der Zoologie übernahm Linné die binäre Nomenklatur erst mit der 10. Auflage des „Systema Naturae" von 1758. In der Mineralogie setzte sich LinNÉs Nomenklatur nicht durch. Insgesamt tragen heute mehr als 12000 Tierund Pflanzenarten hinter ihrem wissenschaftlichen Artnamen ein L., das auf die wissenschaftliche Erstbeschreibung durch Linné hinweist. Darunter ist auch der Mensch, dem Linné den Namen Homo sapiens, der weise Mensch, gab.

Neben seinen systematischen Arbeiten beschäftigte sich LiNNÉ auch mit zahlreichen weiteren naturwissenschaftlichen Themen. Eng verknüpft mit seiner Tätigkeit als Botaniker waren u.a. auch seine gärtnerischen Fähigkeiten. Linné gelang es beispielsweise durch Experimentieren mit verschiedenen Kulturbedingungen, auch sehr seltene exotische Arten in seinen Gewächshäusern zu kultivieren und sogar zu Blüte und Samenreife zu bringen, sodass er auch andere Botanische Gärten mit Pflanzenmaterial versorgen konnte. In diesem Zusammenhang ist zu erwähnen, dass sich LINNÉ zur Überwachung seines Gewächshauses ein zuverlässiges Thermometer wünschte. Als ehemaliger

Abb. 1 (oben): Titelblatt der Species Plantarum.

Abb. 2 (unten): Titelblatt der Systema Naturae. 
Schüler des Mathematik- und Physikprofessors Anders Celsius (1701-1744), ein Neffe seines alten Freundes Olof Celsius, verfolgte er sehr interessiert die Entwicklung der Celsius-Temperaturskala, die durch die zwei Fixpunkte $100^{\circ} \mathrm{C}$ (Gefrierpunkt des Wassers) und $0{ }^{\circ} \mathrm{C}$ (Siedepunkt des Wassers) bestimmt wurde. Die Benennung der Fixpunke hatte Celsius so gewählt, damit Messungen des Wetters ohne lästige Wechsel von Minus und Plus auskommen. LinNÉ war von der neuen Temperaturskala sehr angetan, und verwendete sie sofort. Nach dem Tode Celsius' drehte er die Skala jedoch einfach um. Er erachtete es nämlich für seine biologischen Fragestellungen als sinnvoller, wenn der Gefrierpunkt mit $0{ }^{\circ} \mathrm{C}$ bezeichnet würde, da bei Temperaturen unter dem Gefrierpunkt viele Pflanzen absterben. 1745 ließ er ein erstes Quecksilberthermometer mit dieser neuen Skala $\left(0^{\circ} \mathrm{C}=\right.$ Gefrierpunkt des Wassers, $100^{\circ} \mathrm{C}=$ Siedepunkt des Wassers) bauen, die heute der Standardskala der Temperaturmessung entspricht. Aus Linnés Feder stammt daher auch der weltweit erste Wetterreport mit Temperaturangaben in modernen Grad-Celsius-Werten, die am 16. Dezember 1745 in der Orangerie im Botanischen Garten der Universität Uppsala aufgezeichnet wurden.

Seinen Botanischen Garten in Uppsala, heute auch als LinNé-Garten bezeichnet, legte Linné nach verschiedenen Prinzipien an. Neben systematischen Ansätzen konzipierte LinNé einige Teile der Anlage nach phänologischen Aspekten, die ihn stets sehr interessierten. So weist der Garten nach dem Blühaspekt der dort kultivierten Arten einen Frühlings-, einen Sommer-, einen Herbst- und einen Wintergarten auf. Die tageszeitliche Rhythmik der Blüten zeigte LinNÉ durch eine von ihm entwickelte Blumenuhr. Dabei handelte es sich um eine Blumenrabatte in Form eines Zifferblatts mit zwölf Feldern, die mit den zur jeweiligen Stunde aufblühenden krautigen Pflanzen bepflanzt

Abb. 3 (oben): Buchtitel mit Motiv aus LinNÉs Lapplandreise.

Abb. 4 (unten): Zeichnung einer Eule von LinNé.

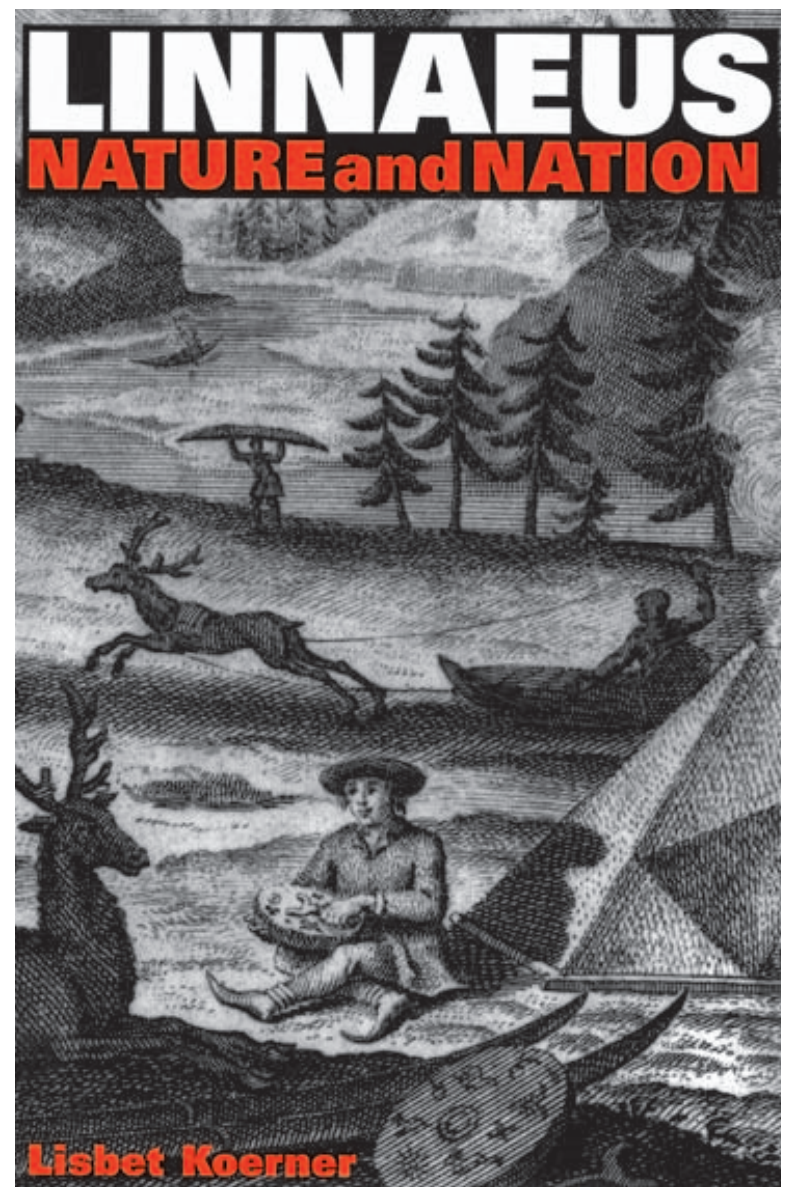

waren. Diese Uhr ermöglichte Linné seinen Angaben zu Folge beim Blick aus seinem Arbeitszimmer in den Botanischen Garten die Uhrzeit auf eine Stunde genau abzulesen.

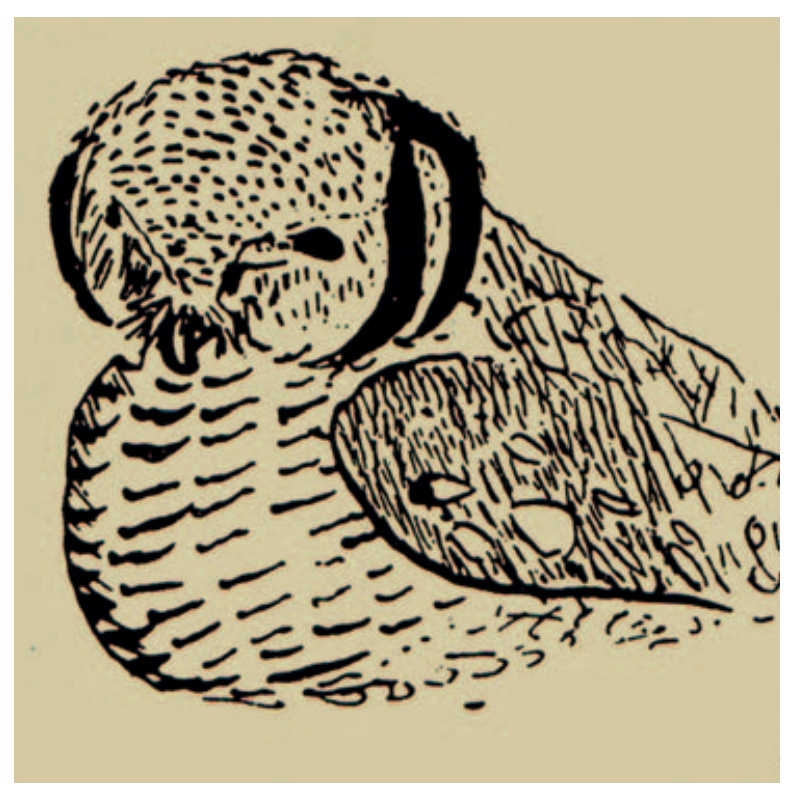




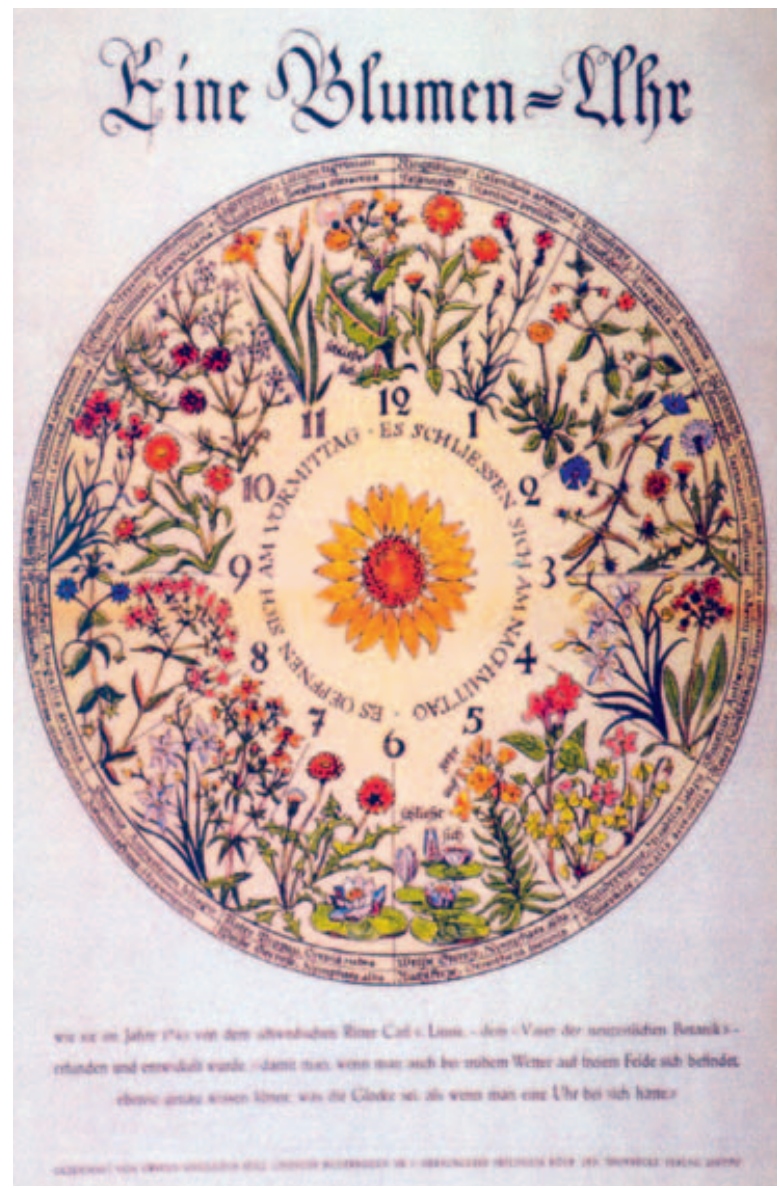

Erste ökologische Ansätze einer Gartengestaltung zeigte LINNÉ, indem er im Botanischen Garten einen Teich anlegen ließ, um seinen Schülern einen Eindruck von der Vergesellschaftung der Wasser- und Sumpfpflanzen geben zu können. Eine Gestaltung von Lebensräumen war zu dieser Zeit eher ungewöhnlich.

\section{Linné als Arzt}

Obwohl er nur über einen kurzen Zeitraum hinweg als praktischer Arzt tätig war, kann durchaus mit Recht gesagt werden, dass LinNÉ auch ein fähiger Arzt war, der allerdings seine eigenen Leiden offensichtlich weniger gut im Griff hatte. Unabhängig von seiner heilenden Tätigkeit beschäftigte sich LINNÉ intensiv mit Volkskrankheiten, Ernährungslehre und - seiner botanischen Neigung entsprechend - mit Arzneimittellehre. So propagierte LinNé beispielsweise eine naturbezogene Lebens- und Ernährungsweise. Viele seiner Ernährungsregeln (u. a. „Diaeta naturalis“ (1733)) könnten einem mo- dernen Gesundheitsbuch entnommen sein und lauten beispielsweise:

- Neugeborene sollen mehrere Jahre lang mit Muttermilch aufgezogen werden

- Branntwein ist Gift

- Tabakrauch, Schnupf- und Kautabak sind Gift

- Alle gekochten Getränke, auch Wasser, sind schlechter als ungekochte

- Alles Übermaß ist schädlich

- Um junge Mütter zum Stillen zu motivieren, wählte Linné bewusst auch den Namen Mammalia für die Gruppe der Säugetiere, zu der er ja auch den Menschen stellte.

In seiner Aufstellung über pflanzliche Heilmittel „Materia Medica“ (1749) hat Linné insbesondere die Bedeutung der heimischen Arzneipflanzen für die Medizin in den Vordergrund gestellt und dabei versucht, auch ihre pharmakologischen Wirkungen nach zehn Geruchs- und zehn Geschmacksarten zu untergliedern. Dies stellt eine durchaus bedeutsame Idee in der Entwicklung der pharmazeutischen Botanik dar. Zudem betonte Linné im Sinne Paracelsus' immer wieder, dass jede Arznei in ihrer Wirkung von der Dosis abhängig ist, und daher vor einer Anwendung genauestens untersucht werden muss.

\section{LINNÉ als Künstler}

Obgleich Linné trotz seines Auslandsaufenthaltes in Holland und seiner Kontakte zu Wissenschaftlern in vielen Teilen der Welt nie eine andere Fremdsprache außer Latein beherrscht hat, wird ihm eine große, fast literarische Fähigkeit Texte zu verfassen zugeschrieben. Insbesondere seine zahlreichen Reisetagebücher und Reisebeschreibungen, die überwiegend auf Schwedisch verfasst wurden, zeichnen sich durch einen einfachen deskriptiven, aber dennoch poetischen Schreibstil aus, der von Johan August STRIndBERG (1849-1912) und anderen Autoren als volksnah und dadurch durchaus bedeutend für

Abb. 5: LinnÉs Blumenuhr. 


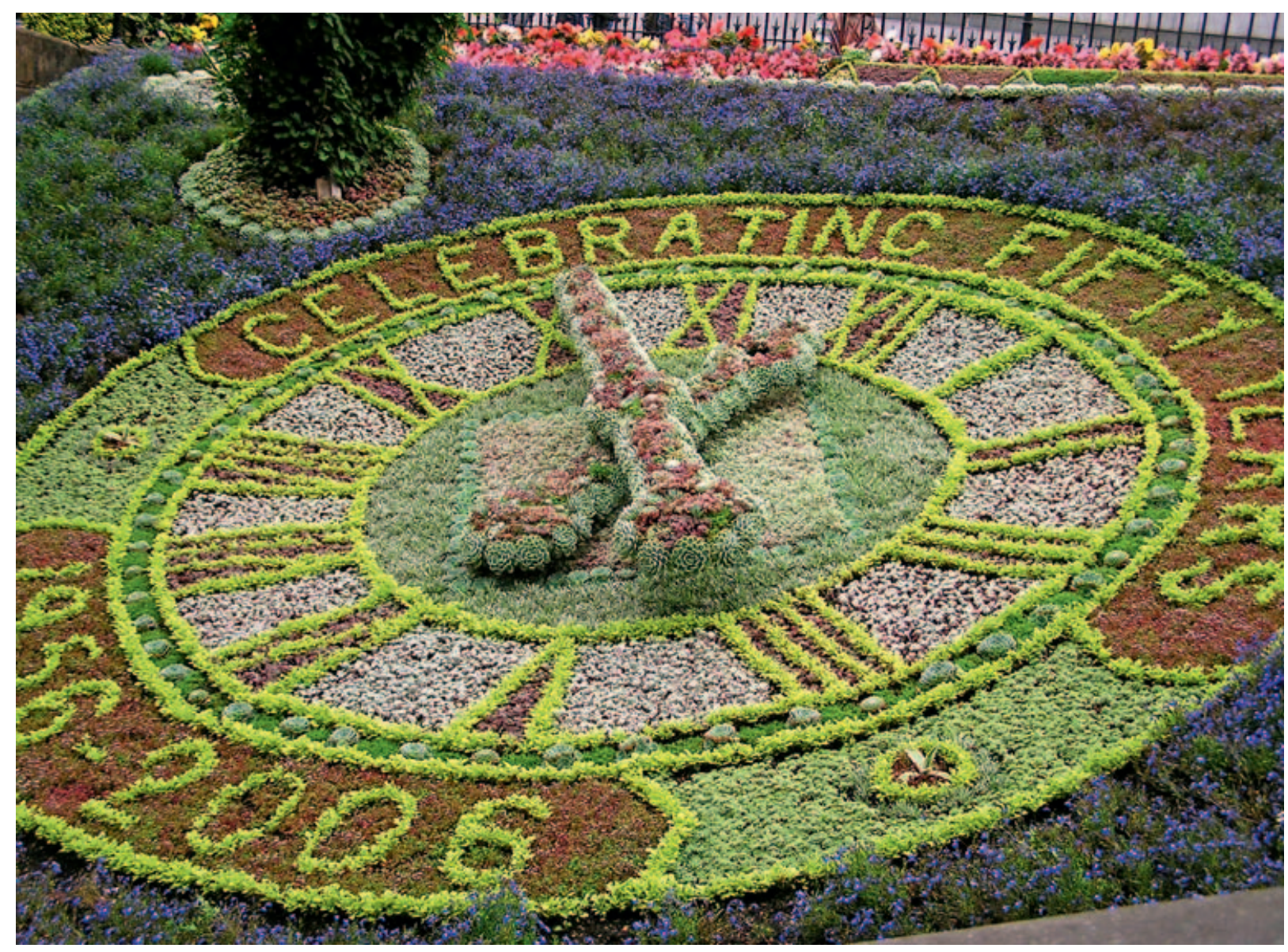

die schwedische Literaturgeschichte angesehen wird. Zudem verfasste LinNé auch Gedichte und bebilderte vor allem seine frühen Manuskripte selbst.

\section{LINNÉ als Mensch}

Aus der zeitlichen Ferne den Charakter eines Menschen zu beschreiben, ist sicher nicht einfach. Quellen, die Linné in seiner Persönlichkeit und seinem Wesen beschreiben, sind selbstverständlich sehr viel seltener als solche, die seine hervorragenden wissenschaftlichen Fähigkeiten wiedergeben. Seine mitreißende Begeisterung für die Naturwissenschaften, sein großer Wissensdurst und seine wertvollen Arbeiten waren es auch, die LiNNÉ in seinem Leben immer wieder gute Freunde finden ließen, die ihn

Abb. 6: Eine moderne Version einer Blumenuhr, die allerdings nicht auf Blühzeiten beruht, in Edinburgh 2006. wie einen Sohn umsorgten. Ohne diese Gönner auf allen seinen Stationen des Lebens hätte LINNÉ sicher nicht das werden können, was er wurde. Dennoch wirkte LinNÉ offenbar auf viele Menschen zumindest nach der ersten Begegnung eher abstoßend. Dies lag wohl vor allem an seiner egozentrischen, überheblichen und teilweise sogar unverschämten Art, die sich aus seiner übermäßigen Selbstüberzeugung von sich und seinen Fähigkeiten ergab. LinNé war sich durchaus bewusst, was er für die Biologie geleistet hatte und wollte dies auch gern gewürdigt wissen. Er sprach selbsteingenommen gerne von sich als „Fürst der Botanik“, ließ sich als „Adam im Paradies“ zeichnen und sagte über sich selbst: „Gott schuf, Linné ordnete“ sowie „Keiner hat mit mehr Eifer seinen Beruf ausgeübt und mehr Hörer an unserer Hochschule gehabt. Kein Naturwissenschaftler hat mehr Beobachtungen in der Natur angestellt. Keiner hat einen solideren Einblick in alle drei Reiche der Natur zugleich gehabt. Keiner war ein größe- 


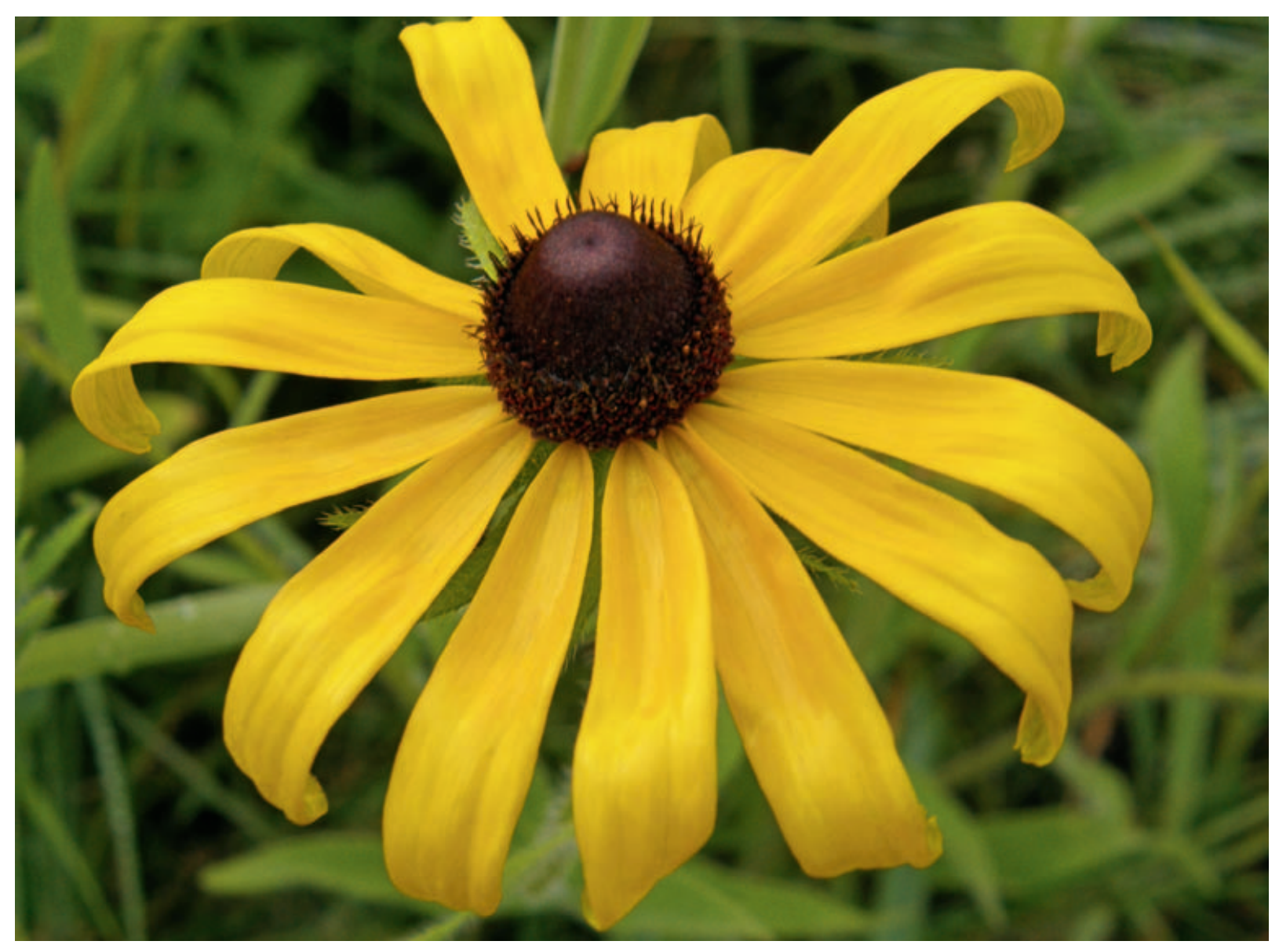

rer Botaniker oder Zoologe. Keiner hat mehr Werke geschrieben, besser, ordentlicher, aus eigener Erfahrung. Keiner so völlig eine ganze Wissenschaft reformiert und eine neue Epoche eingeleitet. Keiner hat eine so über alle Welt ausgedehnte Korrespondenz gehabt. Keiner hat so viele Schüler in so viele Teile der Welt ausgeschickt. Keiner wurde mehr namhaft in aller Welt. Keiner war Mitglied von mehr wissenschaftlichen Gesellschaften ..."

Nicht nur wegen seines überheblichen Auftretens, sondern auch wegen der Inhalte seiner Arbeiten hatte Linné stets auch Gegner. Unter diesen war u.a. der Kollege aus Uppsala NiLs Rosén, der Botaniker Johann Georg SiegesBECK (1686-1755) aus St. Petersburg und der Schweizer Mediziner Albrecht Haller (1708-1777), der LinnÉs Sexualsystem ablehnte und ein natürliches Pflanzensystem forderte. Zudem kritisierte Haller die Willkür, die LinNé in der Tat ans Licht stellte, als er im Zuge seiner neu einzuführenden binären Nomenklatur vertraute Pflanzennamen durch Neuschöpfungen ersetzt. LiNNÉ ging mit seinen Kontrahenten nicht unbedingt sanft um, und konterte bei wissenschaftlichen Angriffen mit Streitschriften und Wortduellen. So heftig seine Feindschaften auch waren, um so intensiver waren aber auch LinNÉs Freundschaften. Besonders mit seinen Schülern stand er in freundschaftlicher Verbundenheit. FABRICIUs berichtet beispielsweise von LINNÉ als einem sehr verständigen, humorvollen Doktorvater, der sehr viel Zeit in die Betreuung seiner Schüler investierte. Auch sollen die meisten der Vorlesungen LinNÉs sehr unterhaltsam gewesen sein, sodass sie nicht nur von Medizinstudenten, sondern auch von Studenten anderer Fakultäten zahlreich besucht wurden. Auch viele berühmte Zeitgenossen Linnés, die

Abb. 7: Rudbeckia hirta, benannt nach LinNÉs Lehrer Rudbeck (1660-1740), Professor der Botanik in Uppsala. 
diesen allerdings zumeist nie persönlich kennen gelernt hatten, äußern sich sehr positiv über Linné. So soll der Philosoph Jean-Jacques Rousseau (1712-1778) LinNÉ ausrichten haben lassen, dass ,ich keinen größeren Menschen auf der Welt kenne“. Und Johann Wolfgang von Goethe (1749-1838) schreibt: „Mit der Ausnahme von Shakespeare and Spinoza kenne ich niemanden unter den Lebenden, der mich mehr beeinflusst hat!" In seiner Heimat Schweden war LinNé zwar ebenfalls mit Ansehen und Ruhm bedacht worden, die jedoch nach seinem Tode schnell zu zerrinnen schienen. So ist auch zu erklären, dass niemand in Schweden Bedenken hatte, als die Witwe LinnÉs seinen gesamten Nachlass ins Ausland verkaufte. Erst Jahrzehnte später wurde LinNé in Schweden wiederentdeckt und markierte nun den Beginn einer schwedischen NaturwissenschaftlerTradition. Der 200. Jahrestag des Geburtstages von LINNÉ im Jahre 1907 wurde bereits gebührend gefeiert, während dies 100 Jahre zuvor nicht der Fall war.

Über LINNÉ als Ehemann und Familienvater weiß man nicht viel. Insgesamt war er ein sehr strenger Vater, obwohl er wegen seiner intensiven Studien der Familie selten zur Verfügung stand. Seinen einzigen Sohn förderte er, seine Töchter durften jedoch nicht die Schule besuchen.

Bei der Neubenennung unzähliger Pflanzenarten im Zuge der Einführung der neuen Nomenklatur ließ sich Linné von der Phantasie beflügeln. Fast alle seiner Freunde, Schüler und Kollegen wurden in Gattungsnamen verewigt, wobei unscheinbare und in Linnés Augen nutzlose Unkräuter die Namen seiner Gegner erhielten. Beispiele für seine Sympathie- und Antipathiebekundungen bei der Namensgebung sind

Abb. 8 (oben): Die Gattung Dablia erinnert an den schwedischen Botaniker DAHL.

Abb. 9 (unten): Bartsia alpina (Alpen-Trauerblume) ehrt den holländischen Naturwissenschaftler JoHANn BARTSCH (1709-1738). Dieser starb in Surinam, wohin er von Hermann BoerhaAve auf Empfehlung von Linné geschickt worden war. Bartsia alpina kommt in Skandinavien, den Alpen und auch auf Island (Foto) vor.
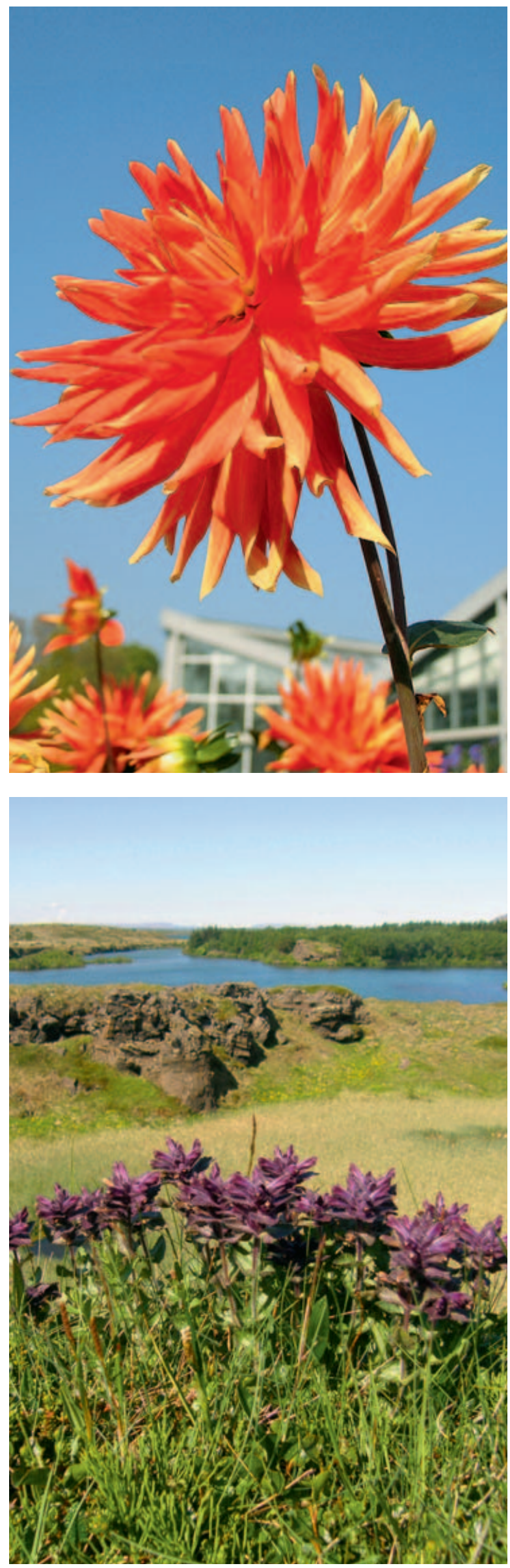


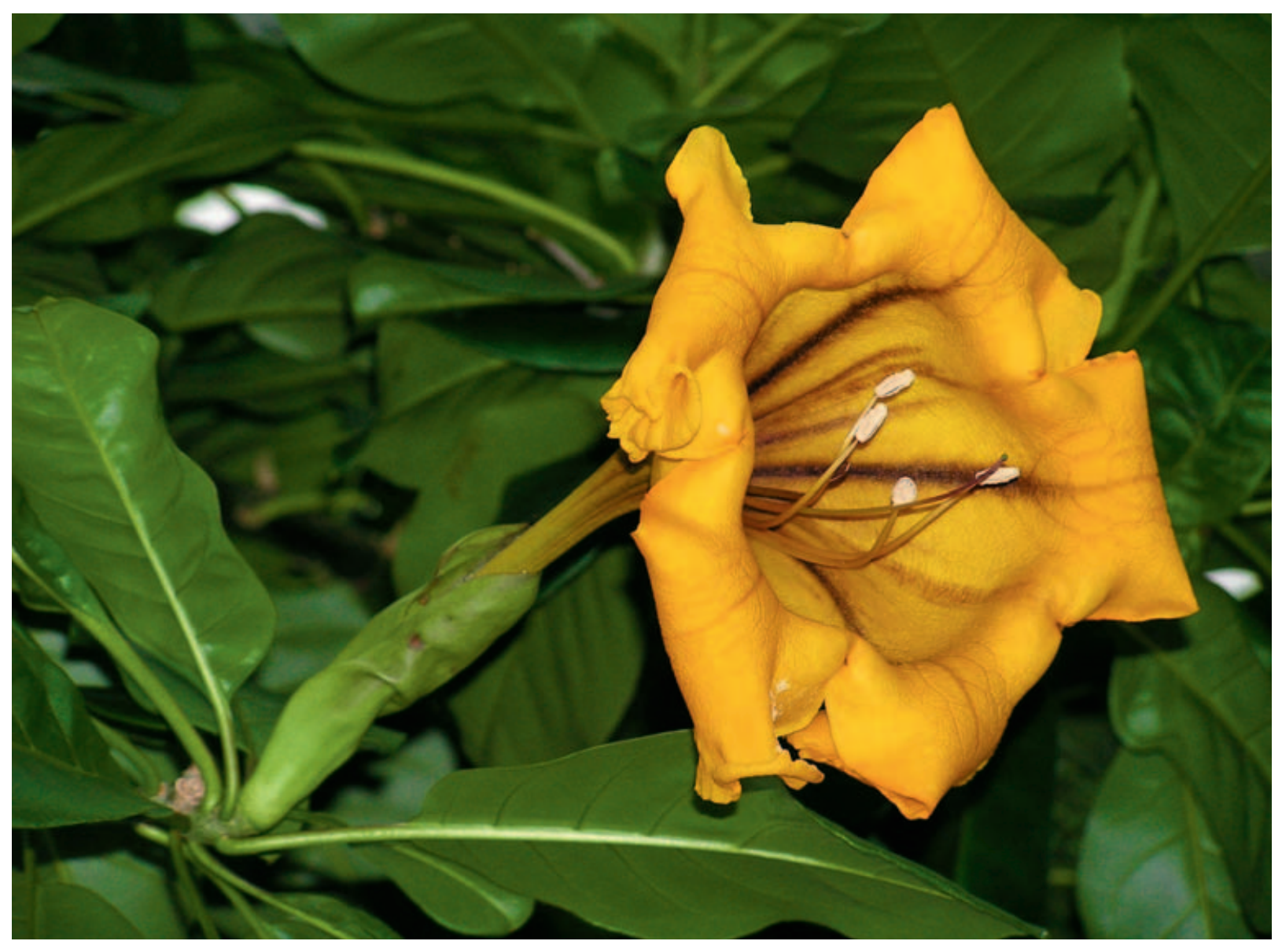

die prächtig blühenden tropisch, subtropisch oder mediterran verbreiteten Gattungen CelsiaVerbascum (nach Celsius), Tournefortia (nach TOURnefort) Rudbeckia (nach RudBeck), Cliffortia (nach CLIFForT) oder Burmannia (nach Burman), die seinen Freunden oder Vorbildern gewidmet sind, während Siegesbeckia (nach Siegesbeck) ein Strauch mit unscheinbaren Blüten ist. Es wird die - allerdings nicht belegbare - Anekdote erzählt, dass LinNÉ einst einen Menschen namens Robert kannte, der durch seinen unangenehmen Körpergeruch auffiel. Daraufhin soll Linné den Stinkenden Storchschnabel, der einen unangenehmen Geruch besitzt, mit dem Namen Geranium robertianum bedacht haben.

\section{LINNÉ und Linnaea}

Aber nun endlich soll verraten werden, wie das Moosglöckchen zu seinem Namen kam. LinNÉ führte bereits eine Regel ein, die auch heute noch bei der Benennung neuer Arten gilt: Ein
Autor, der eine Art entdeckt und neu beschreibt, darf diese nicht nach sich selber benennen. LINNÉ liebte aber das Moosglöckchen, das vor der Reform der Systematik durch Linné als Campanula serpyllifolia beschrieben und somit den Glockenblumen zugeordnet wurde, so sehr, dass er schon zu Beginn der Entwicklung des binären Nomenklatursystems fast bewusst jemanden suchte, der diese Pflanze nach ihm benennen würde. Er fand diese Person in Holland während seines Auslandaufenthaltes in Gestalt von Gronovius, der ihm ja in vielerlei Hinsicht behilflich war. Gronovius gab im Jahre 1737 dem Moosglöckchen den Gattungsnamen Linnaea nach seinem Freund und Schüler LinNÉ. Dieser war darüber sehr glücklich und fügte

Abb. 10: Der Goldkelch (Solandra maxima) wurde nach Daniel Solander (1733-1782) benannt. Zusammen mit dem Botaniker JosepH BANKs nahm er an der ersten Weltumsegelung von JAMEs CoOK (1768-1771) teil. 
gemäß der arktisch-alpinen Verbreitung von Linnaea selbst das Artepitheton borealis hinzu. Später revanchierte sich Linné durch Benennung der tropischen Gattung Gronovia (Loasaceae) nach seinem Freund. Weitere Pflanzengattungen, die später durch andere Autoren zu Ehren Linnés benannt wurden, sind im Übrigen Linnaeobreynia (Capparidaceae), die von LinNé ursprünglich als Breynia bezeichnet wurde und später von HuTCHINSON umbenannt wurde, sowie Linnaeopsis (Gesneriaceae). Und auch die jüngste Schaffung der neuen Pflanzenfamilie Linnaeaceae innerhalb der Dipsacales erinnert an LiNNÉ.

\section{Spuren Linnés und das LinNé-Jahr}

Wie zuvor ausführlich dargestellt, gibt es also auch heute noch zahlreiche Spuren, die uns an Linné und sein Werk erinnern. Ob nun das LinNé'sche System, die binäre Nomenklatur, die zahlreichen von ihm geschaffenen wissenschaftlichen Tier- und Pflanzennamen oder die umgedrehte Celsius-Temperatur-Skala: vieles aus Linnés Arbeit ist heute noch in der Wissenschaft gültig und weit verbreitet. LINNÉs Lieblingsblume ebenso wie seine unzähligen theoretischen Schriften und Abhandlungen, die über 500 bekannten Portraits, Zeichnungen und Standbilder von ihm rufen uns diesen großen Naturforscher in Erinnerung. Wen wundert es, dass auch nach seinem Tode LinNés Name, der Gattungsname Linnaea oder ein Abbild seiner Lieblingsblume als Logo, Symbol oder Name für bedeutende Einrichtungen in Botanik oder Wissenschaft allgemein gewählt wurden. Die Palette reicht hierbei von der traditionsreichen und bedeutenden Linnean Society in London, die ja auch Linnés Nachlass verwaltet, über das Logo des Botanischen Gartens der Memorial University in St. John's,

Abb. 11 (oben): Stinkender Storchschnabel (Geranium robertianum).

Abb. 12 (Mitte): Logo des Botanischen Gartens der Memorial University of St. John's, Neufundland, Kanada. Abb. 13 (unten): Die LinNÉ-Straße in Lund.
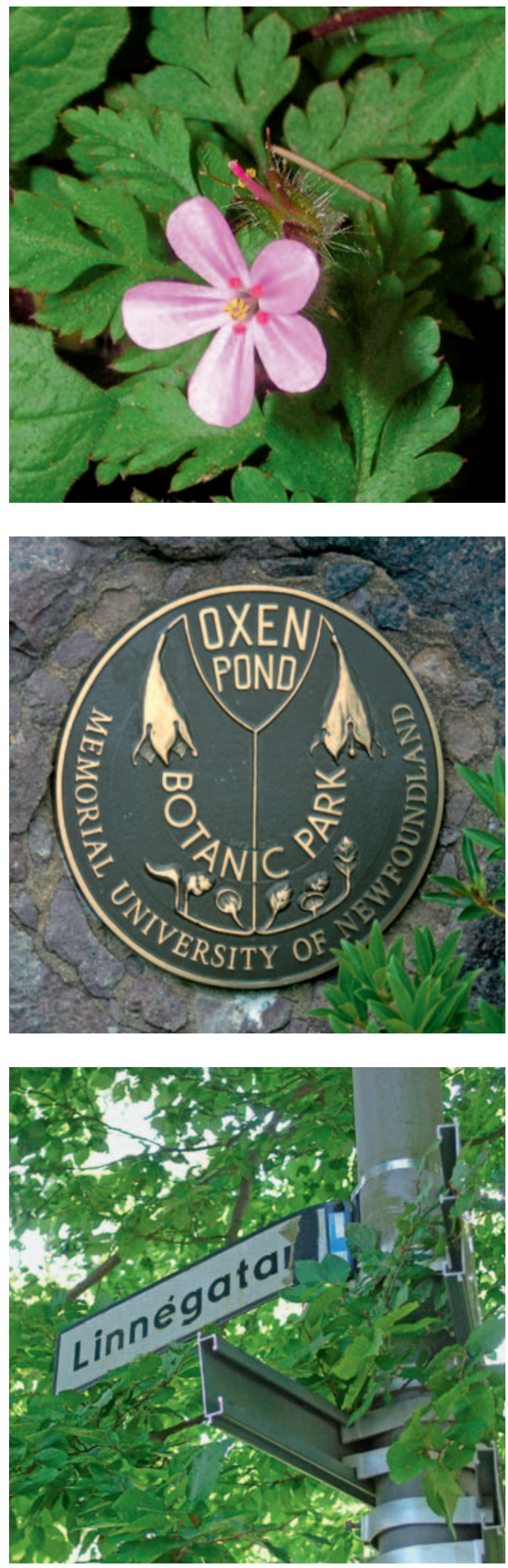


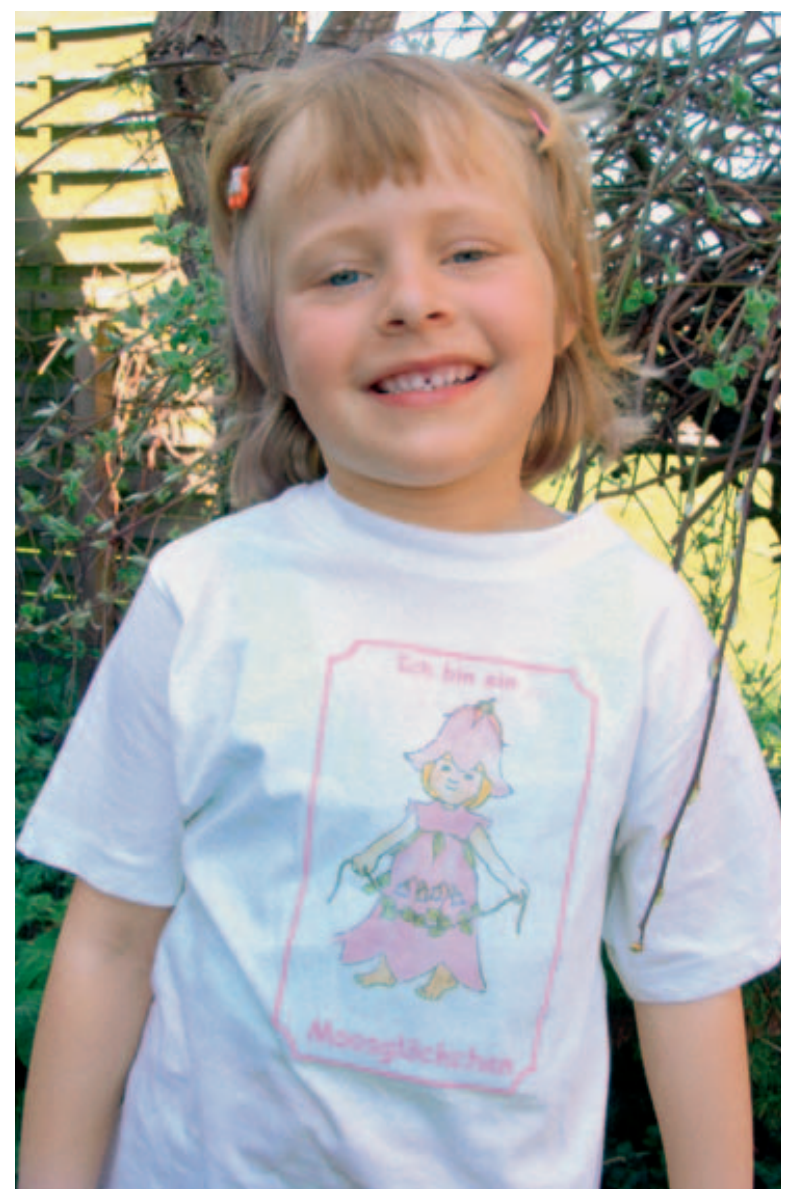

bis hin zum Namen eines modernen pharmazeutischen Unternehmens. Mit der zunehmenden Wertschätzung LinNÉs auch in Schweden entstand dort am Anfang des 20. Jahrhunderts der Mädchenname LinNÉA, der beständig an Popularität zugenommen hat und in den letzten Jahren immer wieder in die "Top ten" der beliebtesten weiblichen Vornamen Schwedens gelangte. So war beispielsweise LinNEA der fünfthäufigste Name neugeborener Mädchen in den Jahren 2002 und 2003, über 60000 Schwedinnen hießen damit im Jahr 2003 LiNNEA. Durch die Sachbilderbuchreihe zu Natur und Kunst vom schwedischen Autorinnenpaar Christina Björk und Lena Anderson, wurde der Name Linnea auch in Deutschland bekannt.
Wer sich heute für LinNé interessiert, sollte nicht verpassen, an seinen Hauptwirkungsort in Schweden zu reisen, nach Uppsala. Hier können der Botanische Garten (LinNé-Garten) und sein Wohnhaus (LinNé-Museum), sein Sommersitz in Hammarby (Museum) sowie sein Grab im Dom besichtigt werden. Im LinNÉJahr 2007 wurden diese immerwährenden Gedenkstätten durch ein reiches Programm an Ausstellungen, Vorträgen und künstlerischen Aktionen ergänzt. Im Rahmen der Festaktivitäten erhielten auch alle bedeutenden Orte, an denen LinNé gelebt, gelernt oder gelehrt hat in Schweden und im Ausland - einen zusätzlichen Hinweis „LinNÉ was here“. Das Logo des Festjahres selbst zeigt eine stilisierte Zeichnung LinNÉs der Staubblätter als Einteilungsmerkmal seines Systems der Pflanzen. Mit den Worten aus einem Gedicht über LiNNÉ von Hedvig CharlotTA NoRdenflycht (1718-1763), einer berühmten schwedischen Schriftstellerin und Zeitgenosssin LinNÉs, sollen diese Gedanken und Geschichten über LinNé enden:

Arise, and hasten, Sweden brightest!

To a noble challenge be spurred,

Without envy Linnaeus honour,

Lest his voice alone be heard!

\section{Literatur}

Goerke, H. 1989: CARL von Linné. Arzt, Naturforscher, Systematiker. - Stuttgart.

\section{Internetseiten}

http://www.univie.ac.at/science-archives/wissenschaftstheorie_2/

http://www.linnaeus.uu.se/online/index-en.html http://www.biologie.uni-hamburg.de/b-online/d01/linne.htm

http://de.wikipedia.org/wiki/Linnea

http://de.wikipedia.org/wiki/Blumenuhr

Abb. 14 (oben): Die Blumenfreundin Linnea Steinecke, 5 Jahre alt, mit ihrem Lieblings-T-Shirt. 